\title{
Introduction to Internet of Everything and the Power of Where Mini-track
}

\author{
Tayfun Keskin \\ Foster School of Business \\ University of Washington \\ Seattle, WA 98195 \\ keskin@uw.edu
}

\author{
Frederick J. Riggins \\ College of Business \\ North Dakota State University \\ Fargo, ND 58108 \\ fred.riggins@ndsu.edu
}

Smart devices connected to the Internet of Things (IoT) platform create new opportunities to provide services to users. Often these smart devices are mobile robots that provide their own power and navigation, or they may be wearables attached to our clothing or person. Examples include autonomous vehicles, robots in factories or homes, and healthcare monitors worn by individuals. As these smart devices move, they can continuously collect data about their changing environment, their own state as they adjust to their environment, or about the user to whom they provide services. Finding ways to collect, store and analyze such big data is a major challenge for service providers. Data from these mobile smart devices also generates a number of privacy challenges that may impact peoples' intention to adopt such devices and services. As these smart devices become more common, how will people adjust to their presence in their everyday lives? How will service providers make use of this data to provide more personalized services? How will our surrounding environment change because of these increasingly ubiquitous devices? Will users be able to exploit this data to become more fulfilled, productive, and happy? Or will the data be used to exploit individuals in ways they may not even know? This mini-track provides a forum for researchers to address these types of issues related to the proliferation of IoT-enabled smart devices and the resulting big data they produce.

The sole paper in this mini-track is entitled "Do Blockchain and IoT Architecture Create Informedness to Support Provenance Tracking in the Product Lifecycle?" by Somnath Mazumdar, Thomas Jensen, Raghava Rao Mukkamala, Robert Kauffman, and Jan Damsgaard. This paper develops and proposes a blockchain-IoT architecture to allay sustainability questions. These days, consumers often lack information about the origin and provenance of the products they buy. They may ask: Is a food product truly organic? Or, what is the origin of the gemstone in the ring I purchased? They also may have sustainability concerns about the footprint of a product at the end of its life. Producers and sellers, meanwhile, wish to know how longitudinal tracking of the provenance of products and their components can boost their sales prices and after-market value, and reveal new business opportunities. The authors of this study focus on how the product lifecycle (PLC) can be leveraged to track information that typically has not been available to support distributed activities. Instead, they have been supported by the manufacturers that create new products. They propose an architecture that utilizes blockchain and the Internet of Things (IoT) to support a range of PLC use case scenarios - from production to marketing and consumption, to maintenance and refurbishment, as well as recycling and disposal. They also offer design thinking about blockchain-IoT architecture to support products such as textiles, furniture and food. A key contribution is an architecture for cross-PLC management support and an explanation of its potential to enhance value through stakeholder informedness.

Eventually the authors propose an architecture for information that documents the product lifecycle based on blockchain and IoT devices attached to physical products. The proposed platform is different compared to other state-of-the-art and commercial tools that aim to capture data across the PLC. Their most important overall contribution is to show how it is possible to map important information that needs to be captured, stored and made accessible for use by PLC stakeholders - wherever they are, and whatever the PLC stage in which they are involved. The approach that they advocate is essentially aimed at reconsidering how to enhance the information endowment, so it is possible to more fully track PLC activities and further integrate stakeholders' legacy systems into a commonly accessible platform.

All in all, we believe the Internet of Everything is an emerging area that will define the future ecosystem of information systems, especially combined with location and other data. We believe this is a ripe area for future research. 